\title{
A microfluidic array with cellular valving for single cell co-culture $\dagger$
}

\section{Jean-Philippe Frimat, ${ }^{a}$ Marco Becker, ${ }^{a}$ Ya-Yu Chiang, ${ }^{a}$ Ulrich Marggraf, ${ }^{a}$ Dirk Janasek, ${ }^{a}$ Jan G. Hengstler, ${ }^{b}$ Joachim Franzke ${ }^{a}$ and Jonathan West ${ }^{* a}$}

\author{
Received 6th July 2010, Accepted 28th September 2010 \\ DOI: 10.1039/c0lc00172d
}

\begin{abstract}
We present a highly parallel microfluidic approach for contacting single cell pairs. The approach combines a differential fluidic resistance trapping method with a novel cellular valving principle for homotypic and heterotypic single cell co-culturing. Differential fluidic resistance was used for sequential single cell arraying, with the adhesion and flattening of viable cells within the microstructured environment acting to produce valves in the open state. Reversal of the flow was used for the sequential single cell arraying of the second cell type. Plasma stencilling, along the linear path of least resistance, was required to confine the cells within the trap regions. Prime flow conditions with minimal shear stress were identified for highly efficient cell arraying $(\sim 99 \%)$ and long term cell culture. Larger trap dimensions enabled the highest levels of cell pairing $(\sim 70 \%)$. The single cell co-cultures were in close proximity for the formation of connexon structures and the study of contact modes of communication. The research further highlights the possibility of using the natural behaviour of cells as the working principle behind responsive microfluidic elements.
\end{abstract}

\section{Introduction}

The orchestrated behaviour of mammalian cells and tissues is achieved by signaling over a range of distances. The most direct communication mode, juxtacrine signaling, involves physical contact between neighbouring cells. Contact modes of communication enable the highly localised coordination of a wide variety of fundamental biological processes, including embryogenesis, tissue regeneration, stem cell differentiation, immune cell activation and cancer biology. Far from being fully understood, cell-cell interactions are a major topic of research, with the modern onus on isolating single cell responses from those of the ensembled population.

To facilitate single cell-cell contact investigations new tools with unprecedented spatial control are required. Microfabricated systems, with cellular and sub-cellular length scale features, are well equipped for the task. Planar micropatterning approaches provide a route to pair neighbouring cell populations. The early work exploited differences in adhesion between cell types with photolithographic-based collagen patterning used to achieve a heterotypic co-culture. ${ }^{1}$ Similarly, heterotypic cellular interfaces can be produced by selectively masking surfaces using microfluidic poly(dimethylsiloxane) (PDMS) stencils ${ }^{2}$ or Parylene $\mathrm{C}$ stencils. ${ }^{3}$ Sequential cell patterning can also be achieved using photoresponsive polymer films ${ }^{4}$ or by using electrochemically active surfaces to modulate ligand presentation. ${ }^{5}$ More recently, cells have been metabolically decorated with DNA

${ }^{a}$ Leibniz_Institut für Analytische Wissenschaften-ISAS-e.V., Otto-Hahn-Str.6b, D-44227 Dortmund, Germany. E-mail: west@isas.de ${ }^{b}$ Leibniz Research Centre for Working Environment and Human Factors at the University of Dortmund (IfADo), Ardeystr. 67, D-44139 Dortmund, Germany

$\uparrow$ Electronic supplementary information (ESI) available: Arrayed particles, HT29 and MCF-7 cells (Fig. S1). Slow single cell arraying video, cell migration (Fig. S2) and on-chip culture for 5 days (Fig. S3). See DOI: $10.1039 / \mathrm{c} 01 \mathrm{c} 00172 \mathrm{~d}$ sequences and patterned as adjacent co-cultures by hybridization with complementary surface-tethered DNA sequences ${ }^{6}$ or directly to cells likewise encoded with the complementary sequence. $^{7}$

Micromechanical methods can also be used to position cells with high precision. A prime example was the use of interdigitated silicon combs for the controlled contact, or separation, of heterotypic populations. ${ }^{8}$ Microfluidics can also be harnessed for reliable single cell handling. Commonly, cells injected within a flow are directed along the fluidic path of least resistance to sub-cellular-sized exits where they become mechanically anchored for analysis ${ }^{9}$ or for high efficiency electroporation. ${ }^{10,11}$ This approach allows the sequential trapping of single cells along a row of microfluidic exits. Using a mirrored configuration, a contacting heterotypic co-culture of spherical cells was established and used to demonstrate the molecular transfer between the cytosolic compartments of paired cells. ${ }^{12}$ Fluidic streamlines can also be used to corral cells into arrays of 2-layer ${ }^{13,14}$ or sievelike ${ }^{15,16}$ microstructured traps for applications requiring high density analysis. For efficient cell pairing and fusion, Skelley and co-workers have invented an ingenious microfluidic structure for first trapping single cells, followed by reverse parking into a twocell-sized trap to be later joined by a second cell type. ${ }^{17}$

The hydrodynamic arraying systems described above are based on differential fluidic resistances, where fluidic streamlines transport single cells into each trap. Once loaded the cell body diverts the streamlines to exclude subsequent cells. Taking this principle further Tan and Takeuchi have developed a linear particle trap array with each trap sequentially connected using a superimposed serpentine microchannel. ${ }^{18}$ Importantly, particles are arrayed with a perfect yield, making it a prime candidate technology for research with rare and precious cells, such as those harvested from biopsy samples. More recently, parallel arrays of trap structures and bypass channels have been used for tracking yeast ${ }^{19}$ and human cell lineages. ${ }^{20}$ In this contribution we introduce a cellular valving principle for use with a Tan and 
Takeuchi microfluidic architecture for highly efficient and reliable single cell coupling.

\section{Concept}

The single cell co-culturing method is based on a cellular valving concept: cells in suspension have a spherical morphology, and following initial adhesion assume a flattened morphology. Positioning a viable cell at an aperture within a microfluidic path can therefore be used to produce a living valve. This principle can be used within a differential fluidic resistance circuit for coupling single cells. We have opted to adapt the Tan and Takeuchi serpentine microfluidic arraying system, with the difference being the use of mirrored traps interfaced by the sub-cellular-sized aperture. The series of apertures produce the linear path. Illustrated in Fig. 1(A), the serpentine flow path has a higher fluidic resistance than the linear path $\left(R_{2}>R_{1}\right)$, producing a higher linear flow rate $Q_{1}$ than the serpentine flow rate $Q_{2}$ :

$$
\frac{Q_{1}}{Q_{2}}=\left(\frac{C_{2}\left(\alpha_{2}\right)}{C_{1}\left(\alpha_{1}\right)}\right)\left(\frac{L_{2}}{L_{1}}\right)\left(\frac{W_{2}+H}{W_{1}+H}\right)^{2}\left(\frac{W_{1}}{W_{2}}\right)^{3}>1
$$

where $W_{1}$ is the aperture width, $W_{2}$ is the channel width, $L_{1}$ is the aperture length, $L_{2}$ is the length of each U-bend channel segment, $H$ is the channel height and $C(\alpha)$ are constants defined by the aspect ratio $(0<\alpha<1)$, and derived from the Darcy friction factor and the Reynolds number. The aperture $C_{1}\left(\alpha_{1}\right)$ value is 78.8 and the microchannel $C_{2}\left(\alpha_{2}\right)$ value is $57.9 .^{18}$

Cell trapping and flattening modulate the value of $R_{1}$ while $R_{2}$ remains constant. The single cell coupling process is as follows: a first cell is transported within the linear bulk flow and trapped at the first aperture. The cell occludes the linear path, increasing

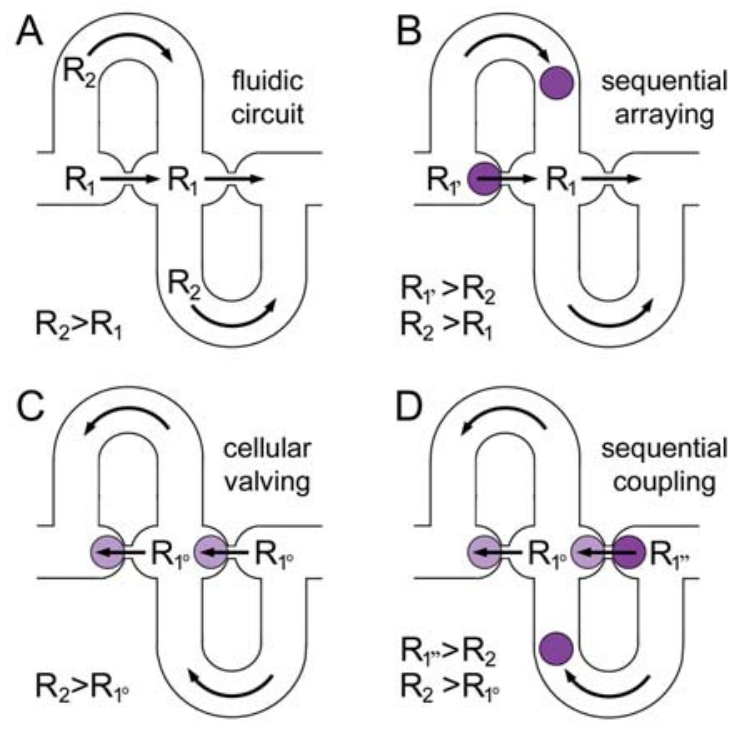

Fig. 1 The cellular valving principle for single cell coupling. The linear path, through the apertures between the mirrored cell traps, has a lower fluidic resistance $R_{1}$ than the serpentine path $R_{2}$ (A). Loading of a spherical single cell (purple) reverses the fluidic resistance ratio, acting to divert subsequent cells $\left(R_{1^{\prime}}>R_{2}\right)$ for sequential $\left(R_{2}>R_{1}\right)$ single cell arraying (B). Viable cells adhere and flatten (lilac), restoring the $Q_{1}>Q_{2}$ condition (i.e. $R_{2}>R_{1}$ 。(C)). Flow reversal is used for introducing a second spherical cell type and sequential single cell coupling $\left(R_{1^{\prime \prime}}>R_{2}\right.$; $R_{2}>R_{1}$ 。(D)). the local fluidic resistance $\left(R_{1^{\prime}}>R_{2}\right)$ and diverting the streamlines. A subsequent cell is diverted into the serpentine pathway for trapping at the second aperture $\left(R_{2}>R_{1}\right.$, see Fig. 1(B)). The process enables the serial arraying of single cells. The cells adhere and flatten, acting as a valve in the open state to restore the $Q_{1}>$ $Q_{2}$ condition ( $R_{2}>R_{1^{\circ}}$, see Fig. 1(C)). The flow is reversed and contains a second cell type for the serial arraying, as before, of single cells within the unoccupied traps (see Fig. 1(D)). The two cell types are in close proximity and can contact one another through the aperture connecting the mirrored traps. In this manner both heterotypic and homotypic single cell co-cultures can be established, with the array format providing the means to couple hundreds of single cell pairs for high throughput screening and the observation of rare events.

\section{Materials and methods}

\section{Design, fabrication and packaging}

The dimensions of the microfluidic circuit are defined by the dimensions of the cell types under investigation. Human SW480 epithelial cells $(\varnothing 14.6 \mu \mathrm{m}, \mathrm{SD} \pm 3.0), \mathrm{MCF}-7$ epithelial-like breast cancer cells $(\varnothing 17.9 \mu \mathrm{m}, \mathrm{SD} \pm 1.5)$ and HT29 colon carcinoma cells $(\varnothing 14.7 \mu \mathrm{m}, \mathrm{SD} \pm 1.1)$ were used in this study. To singularly accommodate these cells, traps with diameters (and typical trap/cell diameter ratios) of $15 \mu \mathrm{m}(\times 1.0), 22 \mu \mathrm{m}(\times 1.5)$, $30 \mu \mathrm{m}(\times 2.0)$ and $37 \mu \mathrm{m}(\times 2.5)$ were designed. The serpentine channel design had a width of $40 \mu \mathrm{m}$, with U-bend segment lengths of $300,500,700$ or $900 \mu \mathrm{m}$ used to provide different flow ratios $(0.6$ to 1.8$)$. The aperture design was $5 \mu \mathrm{m}$ in length and $6 \mu \mathrm{m}$ in width. Channel bifurcations were used to define 8 parallel analysis channels each containing 25 trap pairs (totalling 200 per device).

The microfluidic system was prepared by replica moulding in PDMS (Sylgard 184, Dow Corning) using a $28 \mu \mathrm{m}$ high SU-8 master fabricated by standard photolithographic methods. The resulting PDMS microstructures were documented by SEM imaging (Quanta 200F, FEI, Czech Republic) and are shown in Fig. 2. Ordinarily inlet and outlet vias are prepared by

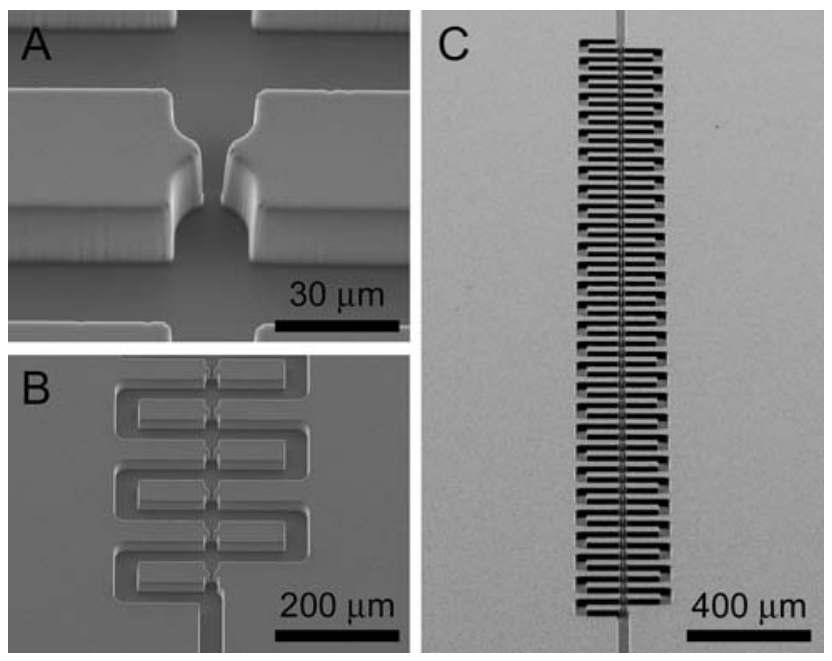

Fig. 2 Microfluidic single cell co-culture array system fabricated by PDMS replica moulding. SEM image of a mirrored pair of single cell traps (A), a series of 6 trap pairs (B) and an entire microfluidic circuit (C). 
puncturing, resulting in large numbers of particle contaminants which present the risk of occluding the fluidic circuit. Instead, we used a frame to align $700 \mu \mathrm{m}$ diameter vertical pins for moulding the inlet and outlet features. The pins were also used to position Luer ports (Scandinavian Biomedical Microdevices, Denmark) for their inclusion within the microfluidic device during thermal curing. To encapsulate the microfluidic structures a thin $(\sim 400 \mu \mathrm{m})$ PDMS layer was first prepared on a glass coverslip support. Plasma treatment $(70 \mathrm{~W}, 40 \mathrm{kHz}$ (Femto, Diener Electronic, Germany)) in a 0.2 mbar oxygen atmosphere for $40 \mathrm{~s}$ was used to activate both surfaces for PDMS-PDMS bonding. Immediately after bonding, media were loaded to preserve the hydrophilic state of the plasma-activated PDMS ${ }^{21}$ and support the adhesion of cells. ${ }^{22}$ Alternatively, bonded devices were left overnight for the diffusive return of oligomers, thereby restoring the hydrophobic state of the PDMS surface, ${ }^{23,24}$ followed by atmospheric pressure air plasma stencilling with a Tesla generator operating with $30 \mathrm{kV}$ at $2 \mathrm{MHz} .{ }^{22}$ By contacting the emitter tip to the microchannel inlet the plasma is routed along the linear path of least resistance, oxidising the PDMS surface to produce a hydrophilic path which supports cell adhesion, while the surrounding PDMS remains biologically inert to confine cell adhesion to the trap regions. Flows were driven by a syringe pump (PHD 2000, Harvard Apparatus) interfaced to the Luer ports via Tygon $^{\mathrm{TM}}$ tubing (ID $1.3 \mathrm{~mm}$ ). For slow continuous flows, $1 \mathrm{~mL}$ pipette tips were inserted into the inlet and outlet Luer ports and used for the hydrostatic pressure-driven delivery of cells and media.

\section{Cell culture and imaging}

Human SW480 epithelial, HT29 colon carcinoma and MCF-7 epithelial-like breast cancer cells were purchased from DSMZ (Germany). Cells were cultured in Dulbecco's modified Eagle medium (DMEM) supplemented with 10\% (v/v) foetal bovine serum (or 10\% (v/v) foetal bovine serum gold (PAA Germany) for the HT29 cell line), 1\% (v/v) Glutamax and 1\% (v/v) penicillin and streptomycin (Sarstedt AG \& Co., Germany). Cells were harvested using $0.25 \%(\mathrm{w} / \mathrm{v})$ trypsin once $80 \%$ confluency was attained. Alternatively cells were harvested and gently disaggregated using accutase (PAA, Germany), a protease and collagenolytic mixture that retains the structural and functional integrity of the cells' surface proteins. The cells were introduced to the microfluidic system and continuously perfused with media by gravity feed during incubation at $37{ }^{\circ} \mathrm{C}$ in a $6 \% \mathrm{CO}_{2}$ atmosphere. The single cell pairing protocol involved loading cells for 30 minutes at $\sim 50 \mu \mathrm{m} \mathrm{s}^{-1}$ using an inlet column height of $25 \mathrm{~mm}$ (15 mm higher than that of the outlet tip). The cells were then incubated for 6 hours for cell adhesion and flattening. The pipette tips were then exchanged and the gravity-driven flow was reversed for loading the second cell type. Single cell co-cultures were incubated for periods up to 5 days with continuous media perfusion at $\sim 5 \mu \mathrm{m} \mathrm{s}^{-1}$ using an inlet column height of $15 \mathrm{~mm}$ ( $5 \mathrm{~mm}$ higher than that of the outlet tip).

Single cell cultures and co-cultures were imaged using an inverted microscope (IX71, Olympus, Germany). Statistical significance was calculated using an independent two-tailed Student's $t$-test. Fluorescent immunostaining targeting connexin $43(\mathrm{Cx} 43)$ was used to observe the connexon gap junctions formed between cell partners. The microfluidic system was first rinsed with a $\sim 50 \mu \mathrm{m} \mathrm{s}^{-1}$ flow of $1 \times$ phosphate buffered saline (PBS) for 10 minutes at $37{ }^{\circ} \mathrm{C}$. Cells were then fixed by the addition of SAV neutral buffered $4 \%$ formaldehyde (Liquid Production, Germany) with room temperature incubation for 1 hour, followed by another $1 \times$ PBS rinse at $\sim 50 \mu \mathrm{m} \mathrm{s}^{-1}$ for 20 minutes and stepwise alcohol dehydration with final equilibration in $1 \times$ PBS. Non-specific binding was blocked using a triple filtered (200 nm pore Ø) solution of $5 \%$ milk/1× PBS for 1 hour at room temperature, followed by a 20 minute rinse at $\sim 50 \mu \mathrm{m}$ $\mathrm{s}^{-1}$. The primary antibody (rabbit anti-Cx43, Dionova, $1: 25$ in $0.3 \% \mathrm{BSA} / 1 \times \mathrm{PBS} / 0.1 \%$ Tween 20 ) was introduced and incubated for 1 hour, followed by $1 \times$ PBS washing $(3 \times 5$ minutes $)$. Goat secondary antibodies labelled with Cy5 (Dionova, $1: 100$ in $0.3 \% \mathrm{BSA} / 1 \times \mathrm{PBS} / 0.1 \%$ Tween 20 ) were then delivered and incubated for 1 hour and washed with $1 \times$ PBS $(3 \times 5$ minutes $)$. Immunostained connexin junctions were documented by fluorescent microscopy with emission detection at $670 \mathrm{~nm}$.

\section{Shear stress simulation}

The CFD flow module of SolidWorks (Dassault Systèmes SolidWorks Corp., MA, USA) was used to simulate the shear stress conditions that the cells experience during microfluidic culture. The highest shear forces are exerted on flat, adherent cells at the microstructured aperture during the loading of the second cell type and during co-culture with media perfusion. The simulation considered the microfluidic dimensions listed above, with a trap diameter of $30 \mu \mathrm{m}$ and a U-bend channel length of $700 \mu \mathrm{m}$. A single adherent cell was modelled with an umbonate morphology, having a diameter of $25 \mu \mathrm{m}$ and rising from zero height at the perimeter to the dome's centre height of $3 \mu \mathrm{m}$. For modelling single cell couples, umbonate pairs were connected via a $5 \mu \mathrm{m}$ long and wide structure. The laminar flow state was described by solving the Navier Stokes equations, using the properties of water and the no-slip condition. For the cell loading simulation the inlet velocity was set to $50 \mu \mathrm{m} \mathrm{s}^{-1}$, and for the coculture perfusion simulation the inlet velocity was set to $5 \mu \mathrm{m} \mathrm{s}^{-1}$. In both cases, an atmospheric pressure $(101 \mathrm{kPa})$ condition was assigned to the outlet of the device. A mesh comprising 800000 uniformly sized rectangular elements was applied with shear stress computations requiring $\sim 7$ hours.

\section{Results and discussion}

\section{Single cell arraying}

Microfluidic arraying using the differential fluidic resistance system was highly reliable and efficient. Shown in Fig. S1(A)†, we have replicated Tan and Takeuchi's result by arraying $20 \mu \mathrm{m}$ diameter monodisperse polystyrene particles. Mammalian cells do not have homogeneous size characteristics and yet were also reliably arrayed with high efficiency ( $\sim 99 \%)$. Arrays of HT29 colon carcinoma cells $(\varnothing 14.7 \mu \mathrm{m}, \mathrm{SD} \pm 1.1)$ and MCF-7 epithelial-like breast cancer cells $(\varnothing 17.9 \mu \mathrm{m}, \mathrm{SD} \pm 1.5)$ are documented in Fig. S1(B and $\mathrm{C}) \dagger$. However, in a preliminary experiment using SW480 epithelial cells $(\varnothing 14.6 \mu \mathrm{m}, \mathrm{SD} \pm 3.0)$ the level of multiple cells per trap was $29.5 \%(\mathrm{SD} \pm 8.6)$. This could be attributed to cell size variations ( $\mathrm{SD} \pm \sim 20 \%$ ), with the smallest cells not adequately occluding the aperture. In addition, 
the trypsin treatment used for cell harvesting did not fully disaggregate the cells. To counter this we instead used accutase, a highly effective tissue dissociation cocktail, which significantly increased $(p<0.02)$ single cell trapping from $69.5 \%(\mathrm{SD} \pm 8.6)$ to $80.6 \%(\mathrm{SD} \pm 4.3)$. Accutase treatment has the further advantage of preserving surface proteins, thereby retaining surface functionality and especially the ability to form cell-cell contacts.

In preliminary experiments, particles and cells were loaded at relatively high velocities ( $\geq 1 \mathrm{~mm} \mathrm{~s}^{-1}$ ) using a syringe pump. However, it was assumed that such velocities within the confines of the microfluidic system would mechanically damage the cells. The arraying method is velocity independent, ${ }^{18}$ presenting the opportunity to operate at lower velocities. Slow cell arraying is documented as a video in the ESI $\dagger$. As a first step, channel bifurcation into 8 parallel channels provides a means to fraction the velocity. To attain much lower velocities the syringe pump was replaced with hydrostatic-driven feed to provide continuous flow (also during incubation) with velocities at the scale of microns per second. Nevertheless, the fluidic restrictions at the trap interface will be the site of highest shear stress for adherent cells during the loading of the second cell type and also during the perfusion of cellular couples. A mean cell loading velocity of $\sim 50 \mu \mathrm{m} \mathrm{s}^{-1}$ and a perfusion velocity of $\sim 5 \mu \mathrm{m} \mathrm{s}^{-1}$ were nominally chosen for experiments, and simulated using SolidWorks to estimate the shear stress experienced by the cells cultured on the microfluidic array. Shear stress scales linearly with the velocity, with maxima occurring at the aperture between the two traps. Presented in Fig. 3, the flow conditions impart shear stresses with maxima of $0.7 \mathrm{dyn}^{-2}$ for 30 minutes during cell loading with a mean velocity of $50 \mu \mathrm{m} \mathrm{s}^{-1}$. This was reduced to $0.07 \mathrm{dyn}^{-2}$ during co-culture perfusion at $5 \mu \mathrm{m} \mathrm{s}^{-1}$. This value is higher than the average $0.025 \mathrm{dyn}^{-2}$ value reported for another microfluidic cell array system, albeit of similar magnitude to interstitial levels and far beneath the physiological shear stress levels experienced by vascular endothelial cells $\left(\sim 10 \mathrm{dyn} \mathrm{cm}^{-2}\right) .{ }^{14}$ It should also be noted that the microfluidic system can be used to impart shear stress gradients across single cells with high precision. Although not the topic of this line of research, this capability could be of great value for investigating cytoskeletal architecture transformations and other cellular responses to shear stress

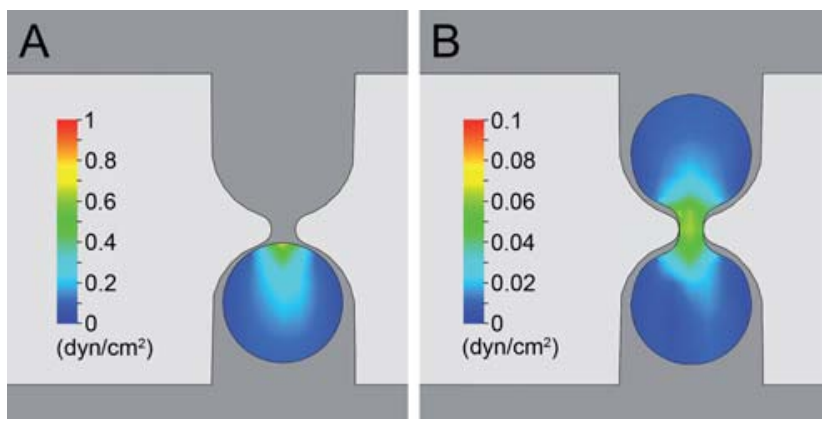

Fig. 3 During secondary cell arraying with a mean velocity of $50 \mu \mathrm{m} \mathrm{s}^{-1}$ the previously arrayed cells experience a moderate shear stress with maxima of $0.7 \mathrm{dyn} \mathrm{cm}^{-2}$ at the interface of the mirrored trap pair (A). Cellular couples experience minimal shear stress (maxima of 0.07 dyn $\mathrm{cm}^{-2}$ ) when perfused with a mean velocity of $5 \mu \mathrm{m} \mathrm{s}^{-1}$ (B). gradients. For example, shear stress is an essential cue required for cardiovascular remodelling during embryogenesis. ${ }^{25}$

A criticism of microfluidic perfusion is the depletion of soluble factors required for paracrine and autocrine signaling. It may be desirable to eliminate these contributions for experiments solely investigating juxtacrine signaling, but for other cases a daily
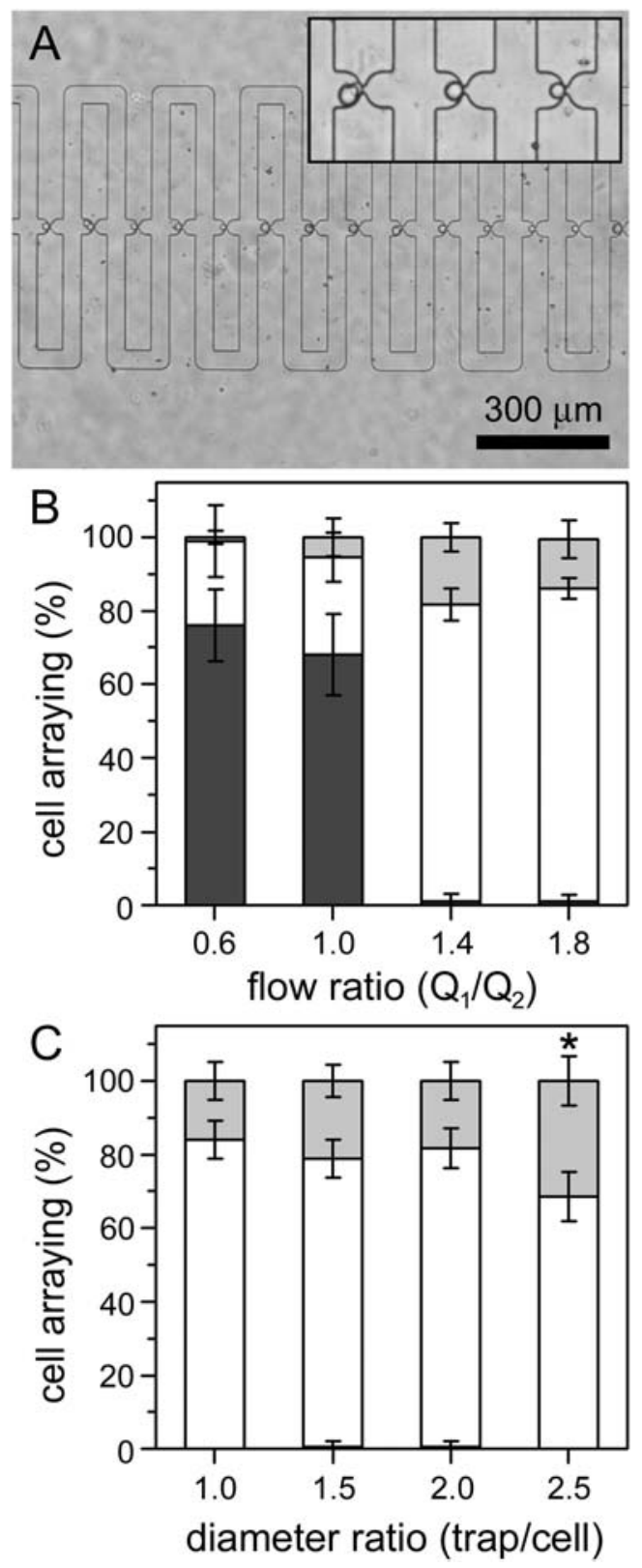

Fig. 4 Highly efficient single cell arraying (A). In this image, 13 single cells are arrayed along with a sole doublet. Inset: enlarged view also demonstrating that the fluidic system was generally insensitive to variations in cell size, with individual trap : cell diameter values ranging from 1.5 to 2.2. The flow ratio significantly impacted the efficiency of single cell arraying (B). Devices with a trap : cell diameter of 2.0 were used. Flow ratios $\leq 1.0$ resulted in large numbers of empty traps (dark grey). Flow ratios $\geq 1.4$ enabled efficient single cell (white) arraying, with few traps containing multiple cells (light grey). The trap diameter had a small effect on single and multiple cell arraying (C). Devices producing a flow ratio of 1.4 were used. 
perfusion for 1 hour at $\sim 5 \mu \mathrm{m} \mathrm{s}^{-1}$ would be sufficient to replenish the media, with soluble factors retained during static incubation for the remainder of the day. In the following proof-of-concept experiments we opted to use continuous media perfusion at $\sim 5$ $\mu \mathrm{m} \mathrm{s}^{-1}$.

The influence of the flow ratio and the trap dimensions was investigated. In this study we used a channel height $(H=28 \mu \mathrm{m})$ double that of the cells' mean diameter $(14.6 \mu \mathrm{m})$. This relatively large size reduces the possibility of shear-induced damage during microfluidic transport to the trap structures. This deviates from the $H<1.4$ cell diameters value recommended by Tan and Takeuchi for use with a flow ratio of $3.95,{ }^{18}$ and demands a lower flow ratio for successful sequential single cell arraying. We examined flow ratios ranging from 0.6 to 1.8 and can confirm the $Q_{1} / Q_{2}>1$ requirement for effective microfluidic arraying. Shown in Fig. 4(A), flow ratios $\geq 1.4$ enabled efficient arraying that was largely insensitive to variations in the size of the SW480 epithelial cells $(\varnothing \mathrm{SD} \pm 3.0 \mu \mathrm{m})$. Recorded in Fig. 4(B), only $\sim 25 \%$ of traps were occupied when using flow ratios $\leq 1.0$, whereas $>98 \%$ of traps were occupied with flow ratios $\geq 1.4$. Single cell occupancy was $80.6 \%(\mathrm{SD} \pm 4.3)$ when using a flow ratio of 1.4 and $85.0 \%$ $(\mathrm{SD} \pm 2.8)$ with a flow ratio of 1.8 . The influence of the trap size was also examined and had only a minor impact on cell arraying characteristics. In this experiment, $>99 \%$ of traps were occupied for trap diameters ranging from $15 \mu \mathrm{m}$ (cell-sized) to $37 \mu \mathrm{m}$. Single cell occupancy was typically $80 \%$, with the exception of the largest traps where only $68.6 \%(\mathrm{SD} \pm 6.7)$ of traps contained single cells. This small but significant reduction $(* p \leq 0.005)$ may be caused by the greater freedom of cell placement with the possibility of failing to divert the streamlines once the first cell is loaded, or that cell doublets were sheltered from flow-induced disaggregation and separation.
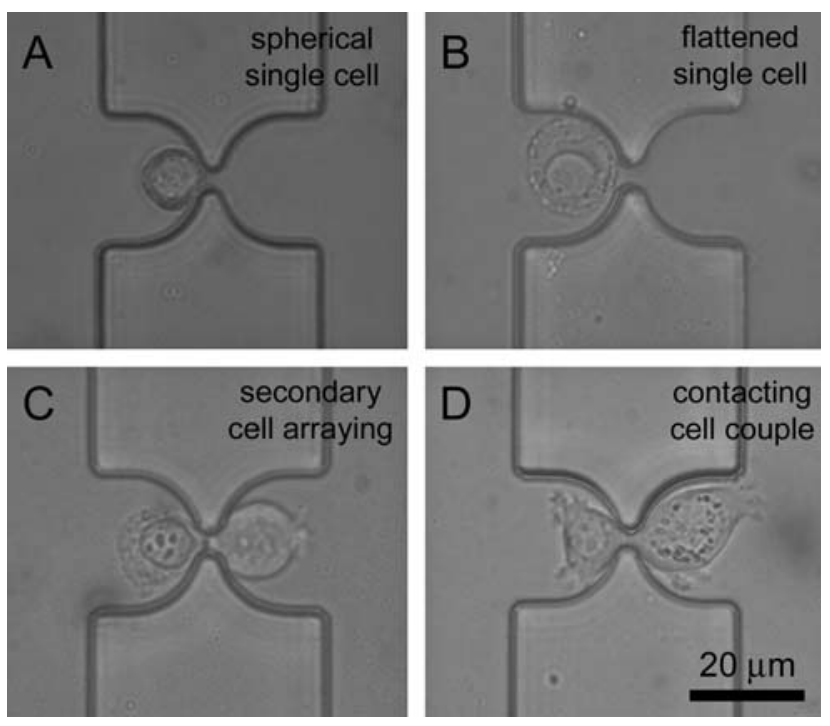

Fig. 5 The cellular valving approach for single cell co-culture. Single cell arraying (A) and cellular adhesion, transforming to a flattened morphology to act as a valve in the open state (B). Introduction of the second cell (C) and following further culture the cell flattened and contacted the first cell (D).

\section{Single cell pairing}

Cell viability is a fundamental pre-requisite for cellular valving. Viable cells can adhere to the substrate and flatten, acting as a living valve to restore the $Q_{1}>Q_{2}$ condition and enable pairing of the second cell type using a reversed flow. The complete single cell pairing process is documented in Fig. 5. Following adhesion, the MCF-7 cells adopted a morphology typical of cells cultured on standard tissue culture substrates (see Fig. 5(B)). This flattened state enabled the delivery of the second cell type which also adhered to the substrate. With continued culture, neighbouring single cell pairs formed physical contacts across the microstructured aperture (see Fig. 5(D)). This demonstrates that the cellular valving principle can indeed be used to establish contacting single cell co-cultures. The method has a wide ranging scope, being suitable for coupling where at least one of the cellular partners can act as microfluidic valve (i.e. adheres and becomes flattened). The coupling principle can also be applied to cell types with different sizes, with the simple requirement that the aperture is smaller than the smallest cell type. Moreover, the use of the differential resistance arraying method eliminates the risk of channel blocking which can occur with systems designed to contact cells across the width of a single microchannel. ${ }^{12}$

Further developments were required to provide a reliable single cell co-culture platform. Dead, non-adherent cells $(\sim 10 \%)$ should be removed. However, flow reversal while viable cells are in the flattened state would only direct dead cells into neighbouring traps immediately across the microfluidic channel. This is solved by exploiting the longer time scales required for cell flattening than initial adhesion. Within 2 hours of loading, viable cells become adherent but retain a relatively spherical morphology. At this stage the cellular valve is in a partially closed state, and by flow reversal dead cells are diverted into the serpentine channel and removed from the system. Following a further 4 hours incubation, the remaining cells flatten in readiness for arraying the second cell type. This method ensures that at least one of the cell partners is viable. With the second arraying phase, $90 \%$ of the cells are estimated to be viable such that $\sim 90 \%$ of the pairs are also likely to contain viable cell partners.

Co-culture experiments require time scales of the order of hours to days. However, with the immediate introduction of aqueous media following plasma-based device assembly, the PDMS maintains a hydrophilic character ${ }^{21}$ which supports cell adhesion $^{22}$ and migration. Within 24 hours some cells migrated outside the trap region (see Fig. S2(A) $\dagger$ ), and to the extremities of the serpentine channel by the fourth day of culture (see Fig. S2(B)†). Migration prevents controlled single cell co-culture. To remedy this limitation we implemented a plasma stencilling method $^{22}$ to pattern cells within the trap regions. This approach requires a hydrophobic PDMS state which can be restored, following plasma bonding, by the diffusive return of oligomers to the surface during overnight incubation in a dry state. ${ }^{24}$ Native PDMS surfaces provide biologically inert backgrounds which resist cell adhesion. ${ }^{26-28}$ Hydrophilic patterns for cell adhesion were provided within the microfluidic system by plasma stencilling using a Tesla generator. ${ }^{22}$ As with the aqueous flows, the plasma was routed along the linear path of least resistance, oxidizing the surface to produce a hydrophilic state which 
supported cell adhesion, while the neighbouring hydrophobic PDMS surfaces confined cell adhesion to the trap regions. Using this approach, $96 \%$ of cells remained within the traps during 48 hours of culture, whereas without plasma stencilling only $76 \%$ remained (a 6-fold loss in occupancy). The increased cell residency significantly increased $(p<0.05)$ the pairing efficiency from $50.3 \%(\mathrm{SD} \pm 11.7)$ to $66.9 \%(\mathrm{SD} \pm 8.5)$. Plasma patterning also acts to increase the $Q_{1} / Q_{2}$ ratio and further promote efficient cell arraying.

To demonstrate heterotypic single cell pairing we have arrayed unlabelled cells with cells labelled with the fluorescent product of calcein AM metabolism. A series of six heterotypic single cell cocultures are shown in Fig. 6(A). An additional experiment was undertaken to identify the formation of gap junctions by labelling the connexin 43 protein within the connexon structures. A routine immunostaining protocol was modified for use within the microfluidic system and involved the use of low flow velocities to prevent the disruption of cellular pairs. Despite the use of this sub-optimal protocol, Cx43 proteins were successfully stained and are documented in Fig. 6(B and C). The image was recorded following 24 hours of co-culture and serves to demonstrate that

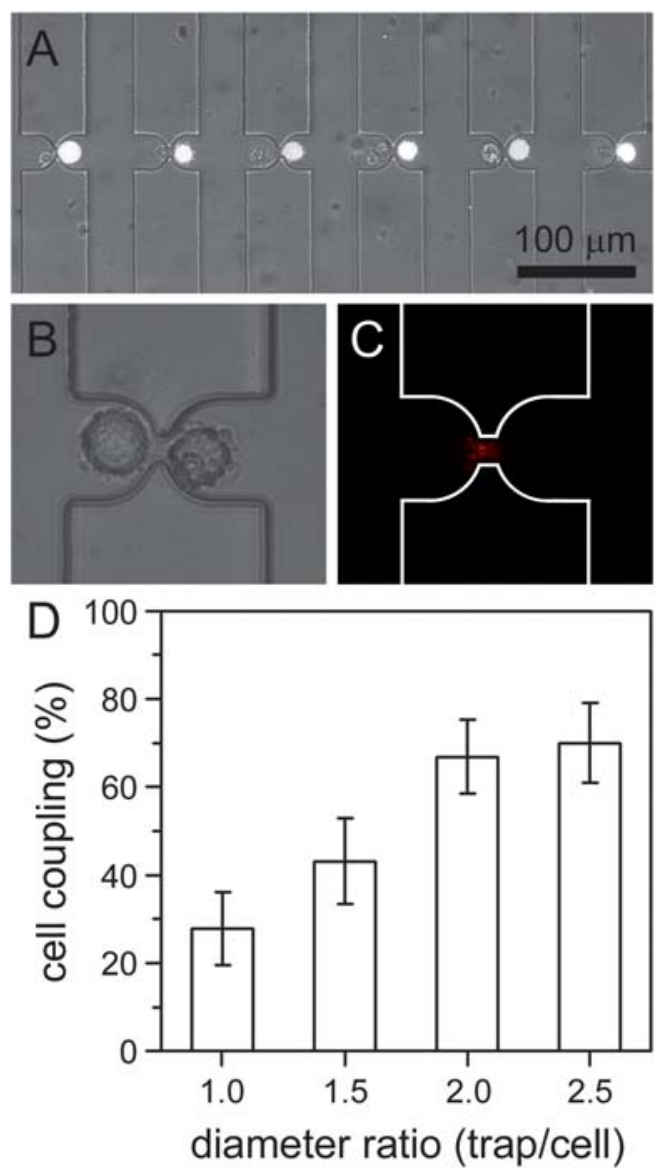

Fig. 6 Heterotypic single cell co-culture. Unlabelled single SW480 cells co-cultured with single SW480 cells metabolically labelled with the fluorescent product of calcein AM (A). A single cell couple (B), and the same couple immunostained with connexin 43 (red, C). A trap : cell diameter ratio of $\geq 2.0$ was required for efficient $(\sim 70 \%)$ cell coupling (D). on-chip conditions are suitable for the maintenance of viable cells and the formation of contact structures necessary for communication. In these experiments $>50 \%$ of cell pairs stained positive for $\mathrm{Cx} 43$ at the cellular interface. However, refinements to the immunostaining protocol are required to determine the levels of Cx43-positive pairs with greater confidence.

The effect of the trap size on cell coupling was also examined. All trap dimensions could be used for cell pairing. As documented in Fig. 6(D), only the larger 30 and $37 \mu \mathrm{m}$ diameter traps could be used to achieve cell pairing rates in excess of $65 \%$, equivalent to the values reported by Skelley and co-workers for their microfluidic reverse parking method. ${ }^{17}$ The largest, $37 \mu \mathrm{m}$ diameter, traps enabled the highest levels of cell pairing with a rate of $70.0 \%(\mathrm{SD} \pm 7.4)$. The trap dimensions are approximately 2.5-fold larger than the spherical cell diameter. In comparison a pairing efficiency of only $27.8 \%(\mathrm{SD} \pm 7.4)$ was obtained with the smallest, cell-sized $(15 \mu \mathrm{m}$ diameter $)$ traps. Our hypothesis is that the smaller traps have insufficient area for complete cellular flattening for valving to render the adjacent trap permissive for arraying of the second cell type.

Cells can be cultured with perfusion in a viable state, with cell cycle continuation resulting in cell division. Shown in Fig. S3†, rapidly dividing HT29 cells proliferate into multi-cell clusters within 5 days of on-chip culture. This provides (additional) homotypic contacts but no longer satisfies the single cell coculture condition. The rate of proliferation thus defines a window for single cell co-culture experiments. With such necessarily short windows of operation biochemically induced cell cycle synchronization is recommended, or the selection of cell cycle sub-populations by fluorescent activated cell sorting (FACS).

\section{Future perspectives}

This paper charts the key features required for the establishment of single cell co-cultures within a differential resistance microfluidic platform. The method could be improved by further optimizing the system dimensions and the inclusion of higher trap numbers for truly high throughput analysis. In addition, problems associated with insufficient cellular disaggregation could be addressed by either using a FACS to select only monodisperse cell collections, or by the integration of an upstream microfluidic method such as pinched-flow fractionation $^{29}$ for the selection of single cell populations prior to arraying. A concern when culturing cells inside microfluidic devices fabricated in PDMS is the loss, by adsorption, of soluble factors secreted by the cells which are necessary for the maintenance of normal cell behaviour. ${ }^{30}$ To overcome this problem, biologically inert glass materials could be used with devices fabricated by methods such as deep reactive ion etching (DRIE).

On-chip analysis is not always practical for many of the large battery of methods available to biologists. The ability to retrieve selected single cells (with knowledge of their individual coculture histories) for off-chip analysis would therefore be a major advantage. In the context of particle-based combinatorial compound libraries this can be achieved by laser-induced microbubble formation for ejecting particles into the flow. ${ }^{18}$ Within our system, the cells could be simply detached from the substrate by incubation in trypsin or accutase. The cells would then adopt a spherical morphology and divert the fluidic 
streamlines along the serpentine channel. The microbubble approach could then be used to simultaneously eject both neighbouring cell partners into the flow for sequential retrieval at the outlet. More straightforward options for cell retrieval are available: again following cell detachment, one cell population type could be sequentially removed by orientating the device for gravity-driven cell retrieval. However, the arraying method first requires further optimization to attain the $100 \%$ pairing yield needed to prevent re-trapping within otherwise vacant traps.

\section{Conclusions}

We have introduced and demonstrated a novel cellular valving concept for use within a differential resistance microfluidic circuit for efficient single cell pairing. The microfluidic conditions support the culture of viable cells and the formation of cell-cell contacts required for intercellular communication. The method is suitable for both homotypic and heterotypic single cell coculture experiments, requires that one or more of the cell types is adherent and can be applied to differently sized cell type combinations. Simple developments are required for assay optimisation and the retrieval of individual cells for experiments requiring off-chip analysis. In summary, the microfluidic arraying technique has great potential to advance co-culture research at the level of a pair of single cells. Beyond this application, the research validates the concept of using the natural behaviour of cells for mechanical operations within microengineered environments.

\section{Acknowledgements}

The authors are grateful to Maria Becker for SEM imaging, Joanna D. Stewart (IfADo) for support with statistical analysis, Wiebke Schormann (IfADo) for providing antibodies, and Sarah Waide and Susanne Funken for cell culture support. The research was financially supported by the German Research Foundation (DFG WE3737/3-1), the Ministerium für Innovation, Wissenschaft, Forschung und Technologie des Landes Nordrhein-Westfalen and the Bundesministerium für Bildung und Forschung.

\section{References}

1 S. N. Bhatia, M. L. Yarmush and M. Toner, J. Biomed. Mater. Res., 1997, 34(2), 189-199.

2 A. Folch and M. Toner, Biotechnol. Prog., 1998, 14, 388-392.

3 D. Wright, B. Rajalingam, S. Selvarasah, M. R. Dokmeci and A. Khademhosseini, Lab Chip, 2007, 7(10), 1272-1279.
4 K. Kikuchi, K. Sumaru, J. I. Edahiro, Y. Ooshima, S. Sugiura, T. Takagi and T. Kanamori, Biotechnol. Bioeng., 2009, 103(3), 552561.

5 M. N. Yousaf, B. T. Houseman and M. Mrksich, Proc. Natl. Acad. Sci. U. S. A., 2001, 98(11), 5992-5996.

6 E. S. Douglas, R. A. Chandra, C. R. Bertozzi, R. A. Matthies and M. B. Francis, Lab Chip, 2007, 7(11), 1442-1448.

7 Z. J. Gartner and C. R. Bertozzi, Proc. Natl. Acad. Sci. U. S. A., 2009, 106(12), 4606-4610.

8 E. E. Hui and S. N. Bhatia, Proc. Natl. Acad. Sci. U. S. A., 2007, 104(14), 5722-5726.

9 M. Yang, C.-W. Li and J. Yang, Anal. Chem., 2002, 74(16), 39914001.

10 M. Khine, A. Lau, C. Ionescu-Zanetti, J. Seo and L. P. Lee, Lab Chip, 2005, 5(1), 38-43.

11 A. Valero, J. N. Post, J. W. van Nieuwkasteele, P. M. ter Braak, W. Kruijer and A. van den Berg, Lab Chip, 2008, 8(1), 62-67.

12 P. J. Lee, P. J. Hung, R. Shaw, L. Jan and L. P. Lee, Appl. Phys. Lett., 2005, 86, 223902.

13 D. Di Carlo, N. Aghdam and L. P. Lee, Anal. Chem., 2006, 78(14), 4925-4930.

14 D. Di Carlo, L. Y. Wu and L. P. Lee, Lab Chip, 2006, 6(11), 14451449.

15 S. Faley, K. Seale, J. Hughey, D. K. Schaffer, S. VanCompernolle, B. McKinney, F. Baudenbacher, D. Unutmaz and J. P. Wikswo, Lab Chip, 2008, 8(10), 1700-1712.

16 S. L. Faley, M. Copland, D. Wlodkowic, W. Kolch, K. T. Seale, J. P. Wikswo and J. M. Cooper, Lab Chip, 2009, 9, 2659-2664.

17 A. M. Skelley, O. Kirak, H. Suh, R. Jaenisch and J. Voldman, Nat. Methods, 2009, 6(2), 147-152.

18 W.-H. Tan and S. Takeuchi, Proc. Natl. Acad. Sci. U. S. A., 2007, 104(4), 1146-1151.

19 A. C. Rowat, J. C. Bird, J. J. Agresti, O. J. Rando and D. A. Weitz, Proc. Natl. Acad. Sci. U. S. A., 2009, 106(43), 18149-18154.

20 S. Kobel, A. Valero, J. Latt, P. Renaud and M. Lutolf, Lab Chip, 2010, 10(7), 857-863.

21 E. Delamarche, A. Bernard, H. Schmid, A. Bietsch, B. Michel and H. Biebuyck, J. Am. Chem. Soc., 1998, 120(3), 500-508.

22 J.-P. Frimat, H. Menne, A. Michels, S. Kittel, R. Kettler, S. Borgmann, J. Franzke and J. West, Anal. Bioanal. Chem., 2009, 395(3), 601-609.

23 J. L. Fritz and M. J. Owen, J. Adhes., 1995, 54(1-2), 33-45.

24 J. Kim, M. K. Chaudhury and M. J. Owen, J. Colloid Interface Sci., 2000, 226(2), 231-236.

25 F. le Noble, V. Fleury, A. Pries, P. Corvol, A. Eichmann and R. S. Reneman, Cardiovasc. Res., 2005, 65(3), 619-628.

26 J.-P. Frimat, J. Sisnaiske, S. Subbiah, H. Menne, P. Godoy, P. Lampen, M. Leist, J. Franzke, J. G. Hengstler, C. van Thriel and J. West, Lab Chip, 2010, 10(6), 701-709.

27 F. Hirschhaeuser, H. Menne, C. Dittfeld, J. West, W. Mueller-Klieser and L. A. Kunz-Schughart, J. Biotechnol., 2010, 148(1), 3-15.

28 H. Hardelauf, J.-P. Frimat, J. D. Stewart, W. Schormann, Y.Y. Chiang, P. Lampen, J. Franzke, J. G. Hengstler, C. Cadenas, L. A. Kunz-Schughart and J. West, Lab Chip, DOI: 10.1039/ c0lc00089b.

29 M. Yamada, M. Nakashima and M. Seki, Anal. Chem., 2004, 76(18), $5465-5471$.

30 M. W. Toepke and D. J. Beebe, Lab Chip, 2006, 6(12), 1484-1486. 